\title{
EL CARMEN DE BOLÍVAR: UNA LECCIÓN QUE NO DEBEMOS OLVIDAR
}

ara nadie es un secreto la creciente preocupación con respecto a la seguridad de la vacunación para el virus de papiloma humano (VPH) en Colombia. Inquietud que tiene su asiento en los recientes hechos ocurridos en el municipio de Carmen de Bolívar ubicado cerca de la costa norte del país, en donde más de 500 niñas que recibieron la vacuna refirieron síntomas que requirieron algún tipo atención médica en el hospital local. La magnitud del evento adquirió tal dimensión que no solamente sobrepasó la capacidad resolutiva de los servicios locales de salud, sino que también demandó el esfuerzo mancomunado de diferentes entidades de índole nacional, departamental y regional con el ánimo apaciguar los ánimos, retornar el orden público a la comunidad y esclarecer los hechos ocurridos (1).

Múltiples teorías han pretendido dar explicación a esta secuencia de eventos, pero son dos las hipótesis sobre las cuales ha recaído principalmente nuestra atención: los síntomas referidos son explicados como una reacción adversa a la vacuna o bien, podría tratarse de un trastorno somatomorfo (respuesta psicógena masiva). El objetivo de la presente editorial es realizar un aporte a la discusión actual mediante la evaluación crítica de la evidencia con respecto a la seguridad de la vacunación para el VPH y efectuar a manera de reflexión, una aproximación acerca de la complejidad del problema ocurrido en el municipio de El Carmen de Bolívar.

Para empezar y con el ánimo de dilucidar lo acontecido, resulta válido preguntarnos: ¿Cuáles son los efectos adversos relacionados con la administración de la vacuna para el VPH? Para responder esta pregunta, me permito recurrir a una actualización de una revisión sistemática de la literatura publicada por el Instituto de Evaluación Tecnológica en Salud de Colombia (IETS) (2), que hace factible identificar dos revisiones sistemáticas $(3,4)$ que fueron consideradas de moderada calidad con el instrumentos Amstar (5) (puntaje 8/11) que analizaron la seguridad de la vacunación para el VPH.

La primera de ellas (3) evaluó la frecuencia de eventos adversos serios en mujeres de 15 a 45 años. La intervención fue la administración de la vacuna bivalente o tetravalente comparada frente placebo o la vacuna para hepatitis A o B. El desenlace de interés fue la frecuencia de cualquier evento adverso serio definido como el hallazgo de cualquier anomalía hematológica o linfática, gastrointestinal, cardiovascular, músculoesquelética o de tejido conectivo, de sistema nervioso central, psiquiátrica, renal, de tracto reproductivo, respiratorio, o el desarrollo de cualquier infección o neoplasia. El seguimiento de los participantes fue de 26 a 41 meses, y el reporte de los eventos adversos se realizó por escrito durante los 15 a 30 primeros días desde la administración de las vacuna y, posteriormente, durante visitas semestrales de seguimiento.

Con base en esta revisión sistemática se pudo establecer que, comparada con el grupo control, la administración de la vacuna para el VPH no se asoció con una mayor frecuencia de eventos adversos serios $(\mathrm{RR}=1,00 ;$ IC 95\%: 0,91-1,09; 7 estudios, 43.856 participantes, $\mathrm{I}^{2}$ : $0 \%$ ). La calidad de la evidencia fue moderada por algunas limitaciones en el riesgo de sesgos en los estudios incluidos. Nótese la precisión de los resultados (IC estrecho, información óptima del tamaño de muestra), la consistencia entre los estudios (baja heterogeneidad) y la aplicabilidad de la infor- 
mación (dos estudios incluidos reclutaron población colombiana) en ausencia de sesgo de publicación.

Por otra parte, la segunda revisión sistemática (4) evaluó la frecuencia de eventos adversos locales o sistémicos posterior a la administración de la vacuna. La población incluida fueron mujeres no embarazadas de 9 a 45 años. De nuevo, la intervención de interés fue el uso de la vacuna bivalente o tetravalente, en tanto que la comparación fue la administración de placebo o de la vacuna para hepatitis A o B. En esta ocasión, el desenlace de interés fue la frecuencia de cualquier evento adverso que pudiese ser catalogado como local (cualquier grado de dolor, eritema o edema en el sitio de punción) o sistémico (presencia de fiebre, fatiga, cefalea, mialgias o artralgias). El seguimiento se realizó durante 7 a 48 meses, y el reporte de los eventos adversos se registró desde la administración de la vacuna y después durante cada visita de seguimiento.

Con base en esta revisión se pudo establecer que la administración de la vacuna para el VPH se asocia con una mayor frecuencia de eventos adversos locales o sistémicos tales como: dolor ( $\mathrm{OR}=3,29$; IC $95 \%$ : 3,00 a 3,60; 6 estudios, 9.427 participantes, $\left.\mathrm{I}^{2}: 19 \%\right)$, eritema (OR = 2,41; IC $95 \%: 2,17$ a 2,68; 5 estudios, 9.133 participantes, $\mathrm{I}^{2}$ : $70 \%$ ), edema $(\mathrm{OR}=3,14$; IC $95 \%$ : 2,79 a 3,53; 5 estudios, 9.133 participantes, $\left.\mathrm{I}^{2}: 78 \%\right)$, fiebre (OR = 1,21; IC $95 \%$ : 1,03 a 1,42; 4 estudios, 8.788 participantes, $\mathrm{I}^{2}$ : $\left.0 \%\right)$, fatiga $(\mathrm{OR}=1,29$; IC $95 \%: 1,18$ a 1,42; 5 estudios, 9.082 participantes, $\left.\mathrm{I}^{2}: 56 \%\right)$, cefalea $(\mathrm{OR}=1,17$; IC $95 \%$ : 1,06 a 1,28; 4 estudios, 8.788 participantes, $\mathrm{I}^{2}: 61 \%$ ), mialgias (OR $=1,97$; IC $95 \%: 1,77$ a 2,20; 4 estudios, 8.013 participantes, $\mathrm{I}^{2}: 57 \%$ ) y artralgias $(\mathrm{OR}=1,40 ;$ IC $95 \%$ : 1,20 a 1,64; 3 estudios, 7.719 participantes, $\mathrm{I}^{2}: 40 \%$ ). La calidad de la evidencia fue baja por algunas limitaciones en el riesgo de sesgos, la presencia de inconsistencia entre los estudios y la imprecisión de los resultados. Esta revisión sistemática no reportó eventos adversos serios.

De esta forma, y a partir de la evidencia disponible, podemos concluir que comparada frente al grupo control, la administración de la vacuna para el VPH parece no incrementar la frecuencia de efectos adver- sos serios (3) pero sí la de efectos adversos menores locales y sistémicos (4).

Ahora bien, al abordar la hipótesis de la respuesta psicógena masiva y luego de revisar algunos artículos disponibles, no resulta para nada lejano recordar lo ocurrido al interior del municipio De El Carmen de Bolívar. En primera instancia es pertinente mencionar que la respuesta psicógena masiva se define como el conjunto de síntomas sugestivos de una enfermedad orgánica para la cuál no existe una causa clara y que se acompaña de una escasa o nula evidencia de enfermedad documentada con el uso de ayudas diagnósticas (6). Se trata de una entidad cuya prevalencia es desconocida, pero que ha sido reportada alrededor del mundo y que involucra personas con síntomas reales los cuales son frecuentemente disparados por una información errónea o mal interpretada (7).

Esta entidad afecta preponderantemente a mujeres durante la infancia y la adolescencia, las cuales exhiben típicamente un cuadro clínico de instauración súbita y de rápida progresión, mostrando un patrón de transmisión bastante corto y que se caracteriza adicionalmente, por el hecho que, otras personas potencialmente expuestas no enferman $(6,7)$. Los síntomas suelen ser precedidos por una exposición comunitaria o ambiental (olor, rumor, toxina reportada) (8) y constan tradicionalmente de cefalea, mareo, dolor abdominal, fatiga, dificultad para respirar, parálisis de una o varias extremidades, ansiedad e incluso pérdida de la conciencia (7-9).

Desafortunadamente, para cuando el brote se ha instaurado y la entidad ha sido finalmente reconocida, el evento ya ha generado un efecto devastador no solamente sobre los individuos afectados sino también sobre la comunidad en general (10). Sea cual sea el desencadenante de esta patología, su reconocimiento oportuno es clave y su abordaje terapéutico complejo; los esfuerzo deben dirigirse a reducir el impacto sobre la población expuesta (6).

Para sobrellevar y finalmente resolver la situación, las comunidades afectadas requieren minimizar su exposición a estimulantes potenciales de ansiedad (despliegue de medios), deben ser notificados a la 
mayor brevedad posible sobre los resultados de los análisis realizados y se les debe proporcionar información clara y veraz que permita disipar los rumores o "causas sospechosas" mediante un diálogo fluido y permanente con los profesionales de la salud $(6,8,10)$.

De esta forma y con base en lo expuesto no resulta difícil entender lo ocurrido. La preocupación no resuelta de padres y pacientes con respecto a la seguridad de la vacuna constituyó el detonante de esta cadena de eventos. La falta de educación, la carencia de una adecuada comunicación y la ausencia de discusión con respecto a los beneficios y riesgos de la vacuna (11), configuró la interior de la población de El Carmen de Bolívar una barrera insalvable $(12,13)$ que llevó al rechazo de la vacuna.

Lo ocurrido en El Carmen de Bolívar nos permitió recordar que si queremos impactar de forma positiva las condiciones de salud, no basta con instaurar medidas seguras y efectivas (14). Debemos educar a nuestras pacientes y sus familias con respecto a los beneficios y los riesgos de las intervenciones (11).

Nos corresponde la obligación de permitir y fomentar la toma informada de decisiones lo que implica decidir con y no por nuestras pacientes. Es probable que solo de esta forma, evitemos a futuro nuevos tropiezos e incrementemos la aceptación de aquellas intervenciones efectivas en salud $(11,12)$.

\section{Carlos Fernando Grillo-Ardila MD, MSc}

Editor Asociado, Revista Colombiana de Obstetricia

y Ginecología

Editor Grupo Cochrane de Infecciones de

Transmisión Sexual

Profesor, Departamento de Obstetricia y

Ginecología,

Universidad Nacional de Colombia

\section{REFERENCIAS}

1. Noticias Caracol. Septiembre 29 de 2014. Disponible en: http://www.noticiascaracol.com/salud/les-danjugo-y-frutas-y-no-hay-solucion-padres-de-ninas-decarmen-de-bolivar

2. Pérez Á, Cañón L, Vanegas E, Olivos J. Evaluación seguridad de la vacuna contra el Virus de Papiloma Humano. Bogotá D.C.: Instituto de Evaluación Tecnológica en Salud IETS; 2014. Visitado 2014 Sept. 15. Disponible en: http://www.iets.org.co/reportesiets/Paginas/Evaluacion-seguridad-vacuna-PapilomaHumano.aspx

3. Lu B, Kumar A, Castellsague X, Giuliano AR. Efficacy and safety of prophylactic vaccines against cervical HPV infection and diseases among women: a systematic review \& meta-analysis. BMC Infect Dis.2011;11:13.

4. Gonçalves AK, Cobucci RN, Rodrigues HM, de Melo AG, Giraldo PC. Safety, tolerability and side effects of human papillomavirus vaccines: a systematic quantitative review. The Brazilian journal of infectious diseases. 2014 S1413-8670(14)00069-5.

5. National Collaborating Centre for Methods and Tools. AMSTAR: assessing methodological quality of systematic reviews. Hamilton, ON: McMaster University. 2011. Visitado 2014 Sept. 15. Disponible en: http://www.nccmt.ca/registry/view/eng/97.html

6. Jones TF. Mass Psychogenic Illness: Role of the Individual Physician. Am Fam Physician. 2000; 62:2649-53.

7. Boss LP. Epidemic hysteria: a review of the published literature. Epidemiol Rev. 1997;19:233-43.

8. Small GW, Feinberg DT, Steinberg D, Collins MT. A sudden outbreak of illness suggestive of mass hysteria in schoolchildren. Arch Fam Med. 1994;3:711-6.

9. Jones TF, Craig AS, Hoy D, Gunter EW, Ashley DL, Barr DB, et al. Mass psychogenic illness attributed to toxic exposure at a high school. N Engl J Med. 2000;342:96-100.

10. Selden BS. Adolescent epidemic hysteria presenting as a mass casualty, toxic exposure event. Ann Emerg Med. 1989;18:892-5. 
11. Fu LY, Bonhomme LA, Cooper SC, Joseph JG, Zimet GD. Educational interventions to increase HPV vaccination acceptance: a systematic review. Vaccine. 2014;32:1901-20.

12. Rambout L, Tashkandi M, Hopkins L, Tricco AC. Self-reported barriers and facilitators to preventive human papillomavirus vaccination among adolescent girls and young women: a systematic review. Prev Med. 2014;58:22-32.
13. Ferrer HB, Trotter C, Hickman M, Audrey S. Barriers and facilitators to HPV vaccination of young women in high-income countries: a qualitative systematic review and evidence synthesis. BMC Public Health. 2014;14:700.

14. Trim K, Nagii N, Elit L, Roy K. Parental Knowledge, Attitudes, and Behaviours towards Human Papillomavirus Vaccination for Their Children: A Systematic Review from 2001 to 2011. Obstetrics and gynecology international. 2012;2012:921236. 